\title{
Wer sagt warum „,nein“ zu Europa? Eine Datenanalyse direktdemokratischer Entscheidungen
}

von Uwe Wagschal

\begin{abstract}
Jüngere Abstimmungen zu Fragen der europäischen Integration machen deutlich, dass Integrationsprojekte immer dann zu scheitern drohen, wenn sie dem Volk zur Entscheidung vorgelegt werden. Mit insgesamt 59 europarelevanten Volksabstimmungen im Zeitraum von 1955 bis 2009 liegt eine hinreichend große Fallzahl vor, um die Determinanten ihrer Ergebnisse zu untersuchen. Basierend auf einem kurzen Überblick zur Ausbreitung direktdemokratischer Verfahren werden sowohl die formalen Beteiligungsregeln (rules in form) als auch die Häufigkeit der Abstimmungen (rules in use) erfasst. Die vorliegenden Daten werden anschließend mit Blick auf zehn unterschiedliche Hypothesen einer multivariaten statistischen Analyse unterzogen. Als signifikant erweisen sich die Faktoren ökonomischer Wohlstand, wirtschaftspolitischer Problemdruck, Intensität des öffentlichen Diskurses im Abstimmungskampf und persönliche Identifikation mit „Europa“.
\end{abstract}

The recent history of referenda on questions of European integration indicates that any push for further integration is in danger of failing if put to a popular vote. Given a set of 59 referenda and similar procedures with European relevance between 1955 and 2009, we command a sufficiently large number of cases to analyse the determinants of their results. Based on a brief overview of the spread of direct democracy across Europe, this contribution details both the formal rules for participation (rules in form) and the frequency of such votes (rules in use). Using ten different hypotheses, the available data are subjected to a multivariate statistical analysis. Significant correlations were found in view of the factors of economic welfare, economic policy pressures, the intensity of public discourse before the vote, and the level of individual identification with "Europe".

\section{Einleitung}

Am 2. Oktober 2009 haben die irischen Bürger ein zweites Mal über den Vertrag von Lissabon abgestimmt, obwohl er bereits im Juni 2008 mit 53,4 Prozent bei einer Stimmbeteiligung von 53,1 Prozent abgelehnt wurde. Die Zustimmung fiel mit 67,1 Prozent eindeutig aus, was partiell auch dem zeitgleichen Höhepunkt der Wirtschafts- und Finanzkrise geschuldet war. So wurde deutlich, dass Europa zu diesem Zeitpunkt den Iren mehr als Rettung denn als Drohung erschien. Aber 
nicht nur der Vertrag von Lissabon, sondern schon das Vorgängerprojekt des europäischen Verfassungswerkes (VVE = Vertrag über die Verfassung Europas) wurde - hier in Frankreich und den Niederlanden - abgelehnt. Nachdem im Anschluss an diese Abstimmungsniederlagen und nach einer „Reflektionsphase“ mit der Einigung auf den Vertrag von Lissabon 2007 vor allem einige symbolische Punkte gestrichen bzw. verändert wurden (Umbenennung des EUAußenministers in „Hoher Vertreter der EU für Außen- und Sicherheitspolitik“, Verzicht auf das Wort „Verfassung“ sowie auf Flagge, Hymne und Europatag), wurden den Iren neben dem Festhalten an der bisherige Zahl der EUKommissare sogar mit einem Zusatzprotokoll einige Garantien und Klarstellungen in Hinblick auf die Abtreibungsfrage, die Neutralität und die Steuerpolitik gewährt. ${ }^{1}$ Die jüngere Abstimmungsgeschichte zu Fragen der europäischen Integration macht damit auf den ersten Blick schon deutlich, dass Integrationsprojekte immer dann in die Gefahr des Scheiterns geraten, wenn sie dem Volk zur Entscheidung vorgelegt werden.

Die jüngsten negativen Volksabstimmungen über die europäische Integration in einzelnen Mitgliedstaaten sind jedoch kein neues Phänomen. Mit insgesamt 59 Volksabstimmungen im Zeitraum von 1955 bis 2009 über Fragen des Beitritts zu europäischen Institutionen, der Ratifizierung europäischer Vertragswerke, notwendiger Verfassungsänderungen oder territorialer Abstimmungen mit Europabezug liegt eine hinreichend große Fallzahl vor, um die Determinanten der Ergebnisse dieser Volksabstimmungen zu untersuchen. Die zentrale Fragestellung dieses Beitrags lautet daher: Gibt es übergreifende und überzufällige erklärende Faktoren der Ergebnisse direktdemokratischer Entscheidungen mit Europabezug? Die Analyse bezieht sich dabei auf die Aggregatebene der einzelnen Staaten. ${ }^{2}$ Nicht (oder nur am Rande) in den Blick genommen werden das individuelle Stimmverhalten auf der Mikroebene ${ }^{3}$ sowie Fragen der Auswirkungen auf

1 Vgl. Europäische Kommission (Hg.): EU-Nachrichten Nr. 2/2009 vom 19.06.2009.

2 Vgl. dazu auch Wagschal, U.: Direktdemokratie und Europäische Integration, in: Freitag, M./Wagschal, U.: Direkte Demokratie - Bestandsaufnahmen und Wirkungen im internationalen Vergleich, Münster, 2007, 40-69.

3 Hug, S.: Voices of Europe, Oxford, 2002; Hobolt, S.B.: Europe in Question, New York, 2009. 
die parlamentarische Demokratie ${ }^{4}$ oder zum vermeintlichen Demokratiedefizit der Europäischen Union. ${ }^{5}$

Der Beitrag gliedert sich wie folgt: Zunächst wird ein kurzer Überblick über die Ausbreitung direktdemokratischer Verfahren in Europa gegeben. Dabei werden sowohl die formalen Beteiligungsregeln (rules in form) als auch die Häufigkeit der Abstimmungen (rules in use) erfasst. Zudem werden die bisher abgehaltenen Volksabstimmungen mit Europabezug überblicksartig zusammengefasst (II.). Daran anschließend werden die Hypothesen formuliert (III.), die die Einflussrichtung der zu untersuchenden unabhängigen Variablen auf der Makroebene (Aggregatebene) angeben. Schließlich werden diese Hypothesen empirisch überprüft (IV.). Das Fazit (V.) fasst die wichtigsten Ergebnisse zusammen und gibt einen Ausblick auf mögliche zukünftige Volksabstimmungen.

\section{Volksabstimmungen in und über Europa - ein Überblick}

Die Funktionslogik direktdemokratischer Entscheidungen ${ }^{6}$ kann an den vier verschiedenen Phasen des politischen Prozesses demonstriert werden: (1) dem input, (2) dem Durchfluss (im Sinne von Eastons ,,throughput“), (3) dem output sowie (4) der Rückkopplung. Dieser Beitrag konzentriert sich besonders auf den in- und output von Volksabstimmungen mit europapolitischem Inhalt.

Der input des politischen Systems hängt dabei stark von der Ausgestaltung der einzelnen direktdemokratischen Elemente ab. Im Gegensatz zu ausschließlich repräsentativ verfassten Systemen sind solche mit direktdemokratischen Beteiligungsformen grundsätzlich „offener“. Das institutionelle set der Beteiligungsmöglichkeiten ist größer, was von Befürwortern direktdemokratischer Entscheidungen mit einer höheren politischen Systemlegitimität verbunden wird. Neben obligatorischen Referenden sind dabei als weitere Instrumente die fakultativen Referenden sowie Plebiszite und Volksinitiativen zu berücksichtigen.

4 Grotz, F.: Direkte Demokratie in Europa. Erträge, Probleme und Perspektiven der vergleichenden Forschung, in: Politische Vierteljahresschrift (PVS) 50/2 (2009), 286-305.

5 Herzog, R./Gerken, L.: Europa entmachtet uns und unsere Vertreter, in: Welt am Sonntag vom 14.01.2007; Kielmansegg, P.G.: Integration und Demokratie, in: Jachtenfuchs, M./Kohler-Koch, B. (Hg.): Europäische Integration, Opladen, 47-72.

6 Möckli, S.: Direkte Demokratie, Bern u.a., 2002; Schiller, T./Mittendorf, V. (Hg.): Direkte Demokratie Forschungsstand und Perspektiven, Wiesbaden, 2002; Vatter, A.: Direkte Demokratie in der Schweiz, in: Freitag, M./Wagschal, U.: Direkte Demokratie - Bestandsaufnahmen und Wirkungen im internationalen Vergleich, Münster, 2007, 71-113. 
Die institutionelle Ausgestaltung direktdemokratischer Entscheidungsverfahren lässt sich anhand verschiedener Kriterien systematisieren. Generell kann dabei nach der Auslösungskompetenz zwischen top-down- oder bottom-up-Instrumenten unterschieden werden. Ferner kann nach dem Verbindlichkeitsgrad von konsultativen oder dezisiven Verfahren ${ }^{7}$ sowie nach dem Abstimmungsgegenstand (z.B. Verfassung, Gesetze, Verträge, Personen) differenziert werden. Topdown-Instrumente sind etwa obligatorische Referenden, die bei einer Änderung der Verfassung automatisch durchgeführt werden müssen. Auch das Plebiszit oder die Volksbefragung, die ein Staatspräsident aus politisch-strategischen Gründen anberaumt, zählt zu diesen top-down-Instrumenten: Obwohl nach der Verfassung oft konsultativ (z.B. in Frankreich), entfalten beide bei erfolgreichem Ausgang einen starken Verbindlichkeitscharakter.

Im Gegensatz dazu ist die Volksinitiative ein bottom-up-Instrument, das die Bevölkerung zur Änderung der Verfassung oder eines Gesetzes lancieren kann. Dabei existieren gewichtige Unterschiede zwischen den konkreten Verfahrensregelungen. So gibt es beispielsweise auch die Möglichkeit unverbindlicher Anregungen (z.B. in Österreich oder auf europäischer Ebene beim Vertrag von Lissabon) oder Volksbegehren, bei denen sich das Parlament mit der Abstimmungsmaterie befassen muss. Das schärfste bottom-up-Instrument ist das fakultative Referendum, welches die Aufhebung von Gesetzen zur Folge hat. Das zentrale Kriterium zur Beurteilung eines Instrumentes als top-down oder bottom-up bleibt freilich dessen auslösende Instanz.

Der Unterschied zwischen beiden Kategorien wird insbesondere beim fakultativen Referendum deutlich. Mit diesem direktdemokratischen Instrument wird ein Gesetz - üblicherweise von der Exekutive eingebracht und von der Legislative verabschiedet - durch eine Unterschriftensammlung von „unten“ angegangen. Entscheidet sich eine Mehrheit gegen dieses Gesetz, so ist es gescheitert; oftmals ist dabei jedoch eine Mindestbeteiligung, ein so genanntes Quorum, erforderlich. Während die Volksinitiative bestimmte Interessen in das politische System einspeist und ihm somit eine besondere input-Funktion innewohnt, ist das fakultative Referendum oft das zentrale Vetoinstrument.

Schließlich können sich Volksabstimmungen nicht nur auf Sachentscheide, sondern auch auf Personen beziehen. Die Direktwahl des Präsidenten oder auch dessen Abberufung (recall) gehören ebenfalls zu den Einrichtungen der Direkt-

7 Schiller, T/Mittendorf, V.: Neue Entwicklungen der Direkte Demokratie, in: Schiller, T./Mittendorf, V., a.a.O., 7-21, hier 14 . 
demokratie, was allerdings in der Fachliteratur nicht einheitlich gesehen wird. ${ }^{8}$ Recall und Direktwahl von Personen werden zu den direktdemokratischen Instrumenten gerechnet, da einerseits das Volk unmittelbar entscheidet und sie andererseits historisch zur Entwicklungsgeschichte direktdemokratischer Instrumente zu zählen sind.

Zusätzliche Beurteilungskriterien direktdemokratischer Instrumente, neben der Auslösungskompetenz, dem Verbindlichkeitsgrad sowie den Abstimmungsgegenständen, sind: (1) Themenbeschränkungen bzw. Themenausschlüsse, (2) Hürden zur Initiierung von Volksentscheiden bzw. Volksinitiativen, (3) Mehrheitserfordernisse (Quoren) bei der Zustimmung (Zustimmungsquorum) bzw. der Beteiligung (Beteiligungsquorum) sowie (4) die Möglichkeit des Parlaments, Gegenvorschläge zu lancieren. Weitere in der Literatur genannte Kriterien sind die Fristen und die Form der Unterschriftensammlung sowie die Regulierung der Finanzierung von Volksabstimmungen. ${ }^{9}$

\section{Rules in form}

Die institutionellen Grundlagen der direktdemokratischen Verfahren (rules in form) in 41 europäischen Ländern werden in Tabelle 1 dargestellt. Die Übersicht basiert auf den aktuellen Verfassungen (Stand: Dezember 2010) und verzichtet auf eine intertemporale Darstellung der Verfassungsentwicklung. Bemerkenswert ist die erkennbare West-Ost-Differenz: Insgesamt weisen die 21 postsozialistischen Untersuchungsländer ein deutlich größeres direktdemokratisches Beteiligungsangebot aus, was auf zwei Faktoren zurückzuführen ist. Zum einen erbrachten die „demokratischen Revolutionen“ in der Neuformulierung der Verfassungen mehr partizipatorische Elemente (Systembruchthese) und zum anderen führte die Orientierung vieler Verfassungen am Semipräsidentialismus Frankreichs zu einer weiten Verbreitung des Plebiszits im post-sozialistischen Raum. ${ }^{10}$

Die am häufigsten vorkommenden direktdemokratischen Instrumente sind auf Basis der Auswertung für alle europäischen Länder: (1) das Plebiszit, (2/3) das fakultative Referendum und die Volksinitiative $(n=21$ für beide) sowie (4) das

8 Ablehnend bei Schiller, T.: Direkte Demokratie, Frankfurt a.M./New York, 2002; zustimmend bei Möckli, S.: Direkte Demokratie, a.a.O.

9 Vgl. Schiller, T.: Direkte Demokratie auf Bundesländer- und Kommunalebene, in: Freitag, M./Wagschal, U. (Hg.): Direkte Demokratie, Münster, 2007, 115-150.

10 Adamovich I.: Entstehung von Verfassungen. Ökonomische Theorie und Anwendung auf Mittel- und Osteuropa nach 1989, Tübingen, 2004. 
obligatorisches Referendum. Weist man jeder der fünf Beteiligungsformen aus Tab. 1 eine „Eins“ zu, dann kommen Serbien, Lettland, Litauen, Rumänien, Slowakei, die Schweiz, Montenegro und die Ukraine auf das größte Partizipationsangebot (Summe: fünf bzw. vier).

Tabelle 1: Institutionelle Grundlagen direktdemokratischer Instrumente (Europa)

\begin{tabular}{|c|c|c|c|c|c|c|}
\hline Staat & 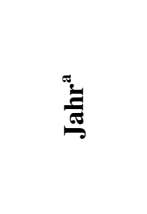 & 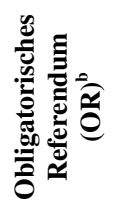 & 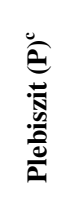 & 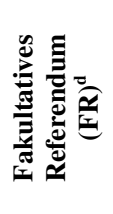 & 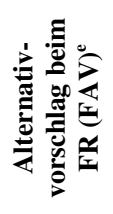 & 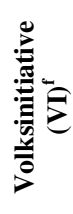 \\
\hline \multicolumn{7}{|c|}{27 EU-Mitgliedstaaten } \\
\hline Belgien & 17.02.1994 & Nein & Nein & Nein & Nein & Nein \\
\hline Bulgarien & 12.07.1996 & Nein & $\mathrm{Ja}$ & Nein & Nein & Nein \\
\hline Dänemark & 05.06 .1953 & $\mathrm{Ja}$ & $\mathrm{Ja}$ & Nein & Nein & Nein \\
\hline Deutschland & 23.05 .1949 & $\mathrm{Ja}^{\mathrm{g}}$ & Nein & Nein & Nein & Nein \\
\hline Estland & 29.06 .1992 & $\mathrm{Ja}$ & $\mathrm{Ja}$ & Nein & Nein & Nein \\
\hline Finnland & 01.03 .2000 & Nein & $\mathrm{Ja}$ & Nein & Nein & Nein \\
\hline Frankreich & 04.10 .1958 & Nein & $\mathrm{Ja}$ & Nein & Nein & Nein \\
\hline Griechenland & 09.07 .1975 & $\mathrm{Ja}$ & $\mathrm{Ja}$ & Nein & Nein & Nein \\
\hline Irland & 22.12 .2001 & $\mathrm{Ja}$ & $\mathrm{Ja}$ & Nein & Nein & Nein \\
\hline Italien & 25.05 .1970 & Nein & $\mathrm{Ja}$ & $\mathrm{Ja}$ & Nein & $\mathrm{Ja}$ \\
\hline Lettland & 06.07 .1993 & $\mathrm{Ja}$ & $\mathrm{Ja}$ & $\mathrm{Ja}$ & Nein & $\mathrm{Ja}$ \\
\hline Litauen & 30.11 .1992 & $\mathrm{Ja}$ & $\mathrm{Ja}$ & $\mathrm{Ja}$ & Nein & $\mathrm{Ja}$ \\
\hline Luxemburg & 19.12.2003 & Nein & $\mathrm{Ja}$ & Nein & Nein & Nein \\
\hline Malta & 21.09 .1964 & Nein & $\mathrm{Ja}$ & Nein & Nein & Nein \\
\hline Niederlande & 05.10 .2004 & Nein & $\mathrm{Ja}$ & $\mathrm{Ja}$ & Nein & Nein \\
\hline Österreich & 01.03 .2010 & $\mathrm{Ja}$ & $\mathrm{Ja}$ & Nein & Nein & $\mathrm{Ja}$ \\
\hline Polen & 17.10.1997 & Nein & $\mathrm{Ja}$ & $\mathrm{Ja}$ & Nein & $\mathrm{Ja}$ \\
\hline Portugal & 02.04 .1976 & Nein & $\mathrm{Ja}$ & Nein & Nein & $\mathrm{Ja}$ \\
\hline Rumänien & 08.12 .1991 & $\mathrm{Ja}$ & $\mathrm{Ja}$ & $\mathrm{Ja}$ & Nein & $\mathrm{Ja}$ \\
\hline Schweden & 01.12 .1994 & Nein & $\mathrm{Ja}$ & Nein & Nein & Nein \\
\hline Slowakei & 01.10 .1992 & $\mathrm{Ja}$ & $\mathrm{Ja}$ & $\mathrm{Ja}$ & Nein & $\mathrm{Ja}$ \\
\hline Slowenien & 23.11 .1991 & Nein & $\mathrm{Ja}$ & $\mathrm{Ja}$ & Nein & $\mathrm{Ja}$ \\
\hline Spanien & 29.12 .1978 & $\mathrm{Ja}$ & $\mathrm{Ja}$ & Nein & Nein & $\mathrm{Ja}$ \\
\hline Tschechien & 01.01 .1993 & Nein & $\mathrm{Ja}$ & $\mathrm{Ja}$ & Nein & Nein \\
\hline Ungarn & 23.10 .1989 & Nein & $\mathrm{Ja}$ & $\mathrm{Ja}$ & Nein & $\mathrm{Ja}$ \\
\hline Ver. Königreich & 30.11 .2000 & Nein & $\mathrm{Ja}$ & Nein & Nein & Nein \\
\hline Zypern & 06.04 .1960 & Nein & Nein & Nein $^{\mathrm{h}}$ & Nein & Nein \\
\hline \multicolumn{2}{|c|}{27 EU-Mitgliedstaaten } & 11 & 24 & 10 & $\mathbf{0}$ & 11 \\
\hline
\end{tabular}




\begin{tabular}{|c|c|c|c|c|c|c|}
\hline Staat & $\frac{\pi}{\tilde{E}}$ & 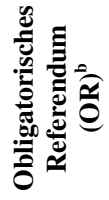 & 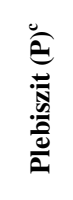 & 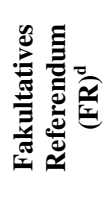 & 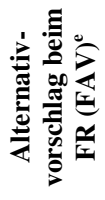 & 己 \\
\hline \multicolumn{7}{|c|}{ Nicht-Mitgliedstaaten } \\
\hline Island & 16.06 .1944 & $\mathrm{Ja}$ & $\mathrm{Ja}$ & Nein & Nein & Nein \\
\hline Liechtenstein & 11.04 .1984 & $\mathrm{Ja}$ & Nein & $\mathrm{Ja}$ & Nein & $\mathrm{Ja}$ \\
\hline Norwegen & 04.11 .1814 & Nein & $\mathrm{Ja}$ & Nein & Nein & Nein \\
\hline Schweiz & 01.07 .1976 & $\mathrm{Ja}$ & Nein & $\mathrm{Ja}$ & $\mathrm{Ja}$ & $\mathrm{Ja}$ \\
\hline Albanien & 21.10 .1998 & Nein & $\mathrm{Ja}$ & $\mathrm{Ja}$ & Nein & $\mathrm{Ja}$ \\
\hline Bos.-Herzegow. & 21.11 .1995 & Nein $^{\mathrm{i}}$ & Nein & Nein & Nein & Nein \\
\hline Kroatien & 22.12 .1990 & Nein & $\mathrm{Ja}$ & $\mathrm{Ja}$ & Nein & Nein \\
\hline Mazedonien & 17.11 .1991 & Nein & $\mathrm{Ja}$ & $\mathrm{Ja}$ & Nein & $\mathrm{Ja}$ \\
\hline Moldawien & 29.07 .1994 & Nein & $\mathrm{Ja}$ & $\mathrm{Ja}$ & Nein & $\mathrm{Ja}$ \\
\hline Montenegro & 19.10.2007 & $\mathrm{Ja}$ & $\mathrm{Ja}$ & $\mathrm{Ja}$ & Nein & $\mathrm{Ja}$ \\
\hline Russland & 12.12 .1993 & Nein & $\mathrm{Ja}$ & $\mathrm{Ja}$ & Nein & $\mathrm{Ja}^{\mathrm{j}}$ \\
\hline Serbien & 29.10 .2006 & $\mathrm{Ja}$ & $\mathrm{Ja}$ & $\mathrm{Ja}$ & $\mathrm{Ja}$ & $\mathrm{Ja}$ \\
\hline Ukraine & 28.06 .1996 & $\mathrm{Ja}$ & $\mathrm{Ja}$ & $\mathrm{Ja}$ & Nein & $\mathrm{Ja}$ \\
\hline Weißrussland & 15.03 .1995 & Nein & $\mathrm{Ja}$ & $\mathrm{Ja}$ & Nein & $\mathrm{Ja}$ \\
\hline \multicolumn{2}{|c|}{14 Nicht-Mitgliedstaaten } & 6 & 11 & 11 & 2 & 10 \\
\hline \multicolumn{2}{|c|}{ Gesamt (alle 41 Staaten) } & 17 & 35 & 21 & 2 & 21 \\
\hline
\end{tabular}

Anmerkungen: $\mathrm{a}=$ Datum, zu dem die aktuell gültigen Regelungen (01.01.2010) zu direktdemokratischen Instrumenten in Kraft traten; $b=$ Rechtlich zwingend vorgesehener Volksentscheid über einen Parlamentsbeschluss; $c$ = Abstimmung, die von der Regierung, dem Staatspräsidenten oder dem Parlament initiiert werden kann; $d=$ Abstimmung über einen Parlamentsentscheid; e = Abstimmung über einen Alternativvorschlag, der im Kontext eines Referendums formuliert wurde; $f=$ Abstimmung über einen vom Volk initiierten Gesetzentwurf; $g$ = Nur im Fall von Länderneugliederungen Art. 29 (2) GG und bei einer Verabschiedung einer neuen Verfassung gem. Art. 146 GG; h = 2004 Plebiszit zur Wiedervereinigung abgelehnt, durch Annan-Plan angeregt; i = Einziges Referendum 1992 extern initiiert; j = Die Volksinitiative wurde im Juni 2004 eingeführt. Datenquellen: Kaufmann, B./Büchi, R./Braun, N.: Guidebook to Direct Democracy in Switzerland and Beyond, 2nd ed., The Initiative \& Referendum Institute Europe, Bern, 2007, 232ff.; Kaufmann, B./Waters, M.D.: Direct Democracy in Europe. A Comprehensive Reference Guide to the Initiative and Referendum Process in Europe, Durham, NC, 2004, 33ff.; eigene Recherchen in den Verfassungstexten. Stand: 01.12.2010.

Das fakultative Referendum, das in der Schweiz ein zentrales Vetoinstrument der Opposition darstellt, existiert ebenfalls in 21 der 41 Untersuchungsländer. Hier besteht ein klare Differenz zwischen Ost- und Westeuropa: Während in nur vier westeuropäischen Staaten (= 20 Prozent dieser Gruppe) eine solche Abstimmungsform möglich ist, besteht diese Möglichkeit in 17 osteuropäischen Ländern (= 81,0 Prozent dieser Gruppe). Und von den vier westeuropäischen Fällen 
zählen nur Italien (mit dem abrogativen Referendum) und die Niederlande zur Europäischen Union - ein bemerkenswerter Befund.

Die unterschiedlichen Formen der Volksinitiative, die entweder auf eine Verfassungsänderung, die Implementierung eines neuen Gesetzes oder eine Parlamentsanregung abzielen, existieren ebenfalls in 21 der 41 untersuchten Staaten. Auch hier besteht wiederum eine deutliche Differenz zwischen der formalen Anwendungshäufigkeit in West- (sechs Staaten bzw. 30 Prozent der Gruppe) und Osteuropa (15 Staaten beziehungsweise 71,4 Prozent der Gruppe). Eine generell hohe Anwendungshäufigkeit ist in Osteuropa, der Schweiz und Liechtenstein zu verzeichnen.

Das obligatorische Referendum (17 von 41 Ländern) weist dagegen keine Unterschiede im Vorkommen zwischen beiden Ländergruppen auf. In Westeuropa kommt es in 40 Prozent und in Osteuropa in 42,9 Prozent der Staaten zur Anwendung.

\section{Rules in use}

Direktdemokratische Volksabstimmungen haben seit den 1970er Jahren eine deutliche Zunahme erfahren. In der Anwendungshäufigkeit (rules in use) gibt es wiederum deutliche Unterschiede in zeitlicher Hinsicht als auch zwischen der Gruppe der EU-Mitglieder und den Nichtmitglieden sowie zwischen west- und osteuropäischen Ländern. Dies gilt sowohl für die Gesamthäufigkeit als auch in der Anwendung einzelner Instrumente.

Von den 1.055 dokumentierten nationalen Volksabstimmungen im Zeitraum von 1848 bis 2010 entfallen 225 auf den Zeitraum vor 1945, 137 auf die Periode von 1945 bis 1970, 229 auf den Zeitraum 1970 bis 1990 und somit 464 auf den Zeitabschnitt nach 1990 (für insgesamt 41 Länder). Dabei ist der deutliche Anstieg über die Zeit nur in geringem Maße den neu entstandenen Staaten zuzuschreiben. Legt man nur die 41 Länder aus Tab. 1 der Auswertung zu Grunde, dann sind für die Periode von 1990 bis 2010 die Spitzenreiter unter den Ländern Europas die Schweiz, Italien, Liechtenstein sowie Litauen (vgl. Abb. 1). Mit dem Hinzutreten der mittel- und osteuropäischen Staaten (MOE) wurden auch hier verstärkt direktdemokratische Instrumente angewendet - oft mit strategischer Motivation. Erweitert man die Rangliste, dann sind unter den zehn Ländern mit den häufigsten Volksabstimmungen sechs und unter den Top-15-Ländern immerhin neun MOE-Staaten. $\mathrm{Zu}$ beachten ist bei diesem Vergleich, dass nur nationalstaatliche Referenden in den Blick genommen werden. Im Hinblick auf die Häufigkeit 
regionaler und lokaler Referenden belegt etwa die Bundesrepublik Deutschland im EU-Vergleich sogar einen Spitzenplatz. ${ }^{11}$

Abbildung 1: Häufigkeitsverteilung direktdemokratischer Entscheidungen in Europa von 1990 bis 2010

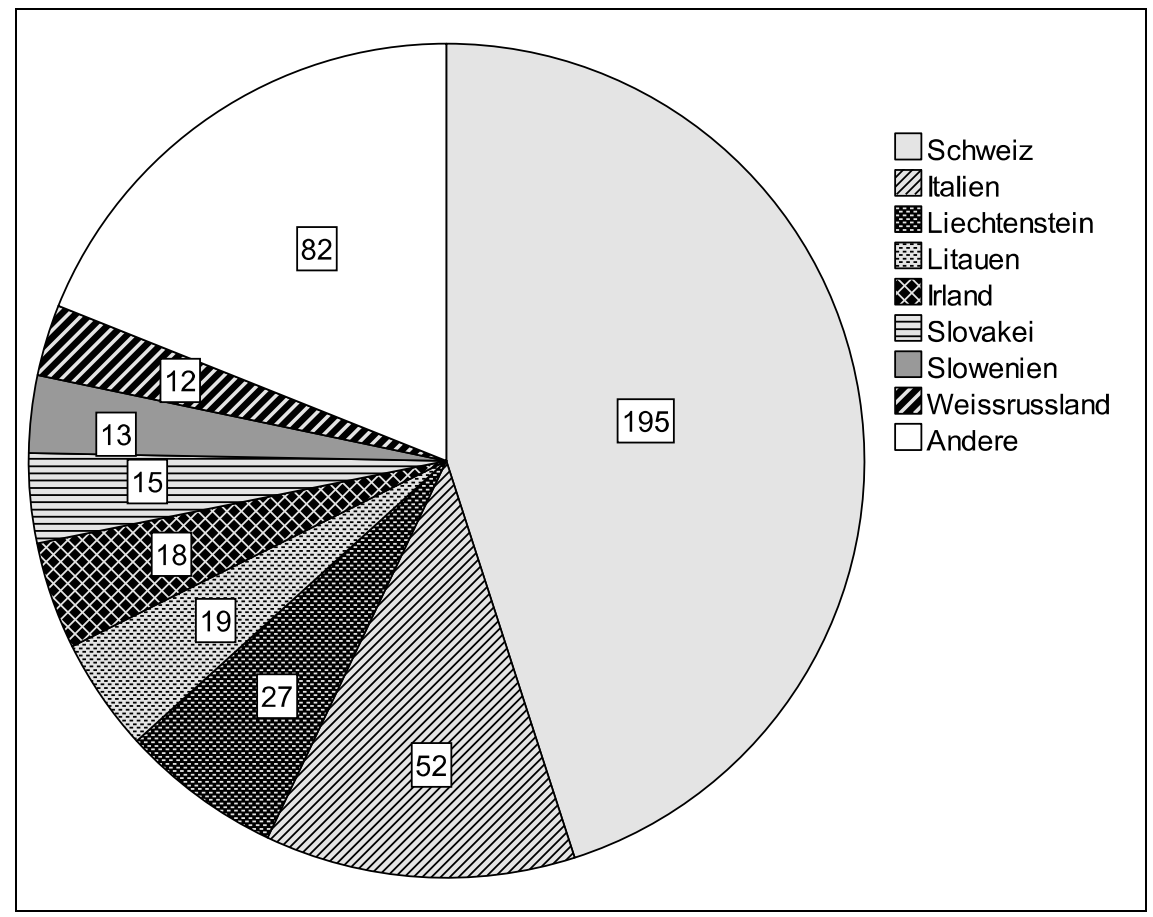

Anmerkung: Die Daten beruhen auf den Auswertungen nationaler Statistiken sowie auf der Datenbank des Research Centre on Direct Democracy (C2D) an der Universität Genf (http://c2d.unige.ch) und der Datenbank ,Suchmaschine für direkte Demokratie“ (http://www.sudd.ch).

Von Relevanz - auch für die folgende Analyse der Volksabstimmungen mit europäischem Inhalt - ist die Frage, ob es einen Zusammenhang zwischen den rules in form und den rules in use gibt. Die formalen Beteiligungsregeln (rules in form) sind über die Summe der Beteiligungsmöglichkeiten aus Tab. 1 operationalisiert, während die rules in use, also die Häufigkeit der Abstimmungen, über die Zahl der Volksabstimmungen erfasst werden (vgl. Abb. 1). Die Rangkorrelationen der rules in use mit den Beteiligungsmöglichkeiten erweist sich in allen

11 Walter-Rogg, M.: Direkte Demokratie, in: Gabriel, O./Kropp, S. (Hg.): Die EU-Staaten im Vergleich, Wiesbaden, 2002, 236-267, hier 258. 
41 Ländern als mittelstark bis stark $\left(r_{s}=0,59\right)$. Ein höheres formales Beteiligungsangebot führt somit auch zu einem höheren Gebrauch der einzelnen direktdemokratischen Instrumente.

Insgesamt konnten 59 Volksabstimmungen nach 1945 mit einem Europabezug identifiziert werden. Die erste europarelevante Volksabstimmung nach dem Zweiten Weltkrieg fand im Oktober 1955 im Saarland statt und hatte dessen Europäisierung zum Gegenstand, ist im Prinzip aber als nicht-nationalstaatlich zu bewerten. Zudem gab es drei europarelevante Volksabstimmungen in zwei außereuropäischen Gebieten/Ländern (Grönland und Aserbaidschan, hier sogar zwei Abstimmungen) sowie zwei weitere subnationale Abstimmungen auf den Åland-Inseln in Finnland (1994). Eine weitere Europaabstimmung - zum Amsterdamer Vertrag - wurde in Portugal 1998 durch das Verfassungsgericht vor der Abstimmung annulliert, da die Fragestellung „nicht klar und präzise“ formuliert war. ${ }^{12}$ Schließt man die subnationalen Fälle aus der nachfolgenden Analyse aus, bleiben insgesamt 54 Entscheide übrig. Diese Fallzahl wird sich in der Regressionsanalyse - auf Grund der Datenverfügbarkeit und inhaltlicher Entscheidungen - noch etwas reduzieren (vgl. unter IV.).

Unter diesen 54 Volksabstimmungen sind 13 Volksabstimmungen (24,1 Prozent) mit einem negativen Ausgang für die europäische Integration, während 41 ein integrationsförderndes Ergebnis aufwiesen (75,9 Prozent). Betrachtet man nur die Abstimmungen von EU-Mitgliedstaaten zum Zeitpunkt der Abstimmung (23 Fälle), also der insider, dann liegt die Ablehnungsrate bei 30,4 Prozent (sieben Fälle). Die sechs ablehnenden Entscheide der outsider, also der Länder außerhalb der EU, haben jeweils spezifische, vor allem ökonomische Erklärungsgründe, etwa im Falle Norwegens (zwei ablehnende Abstimmungen) und der Schweiz (drei ablehnende Volksentscheide). So begründete Anne Enger Lahnstein (der Kopf der EG-Gegner 1994 in Norwegen) ihr „Nein“ auch damit, dass das Land es sich wegen seines Ölreichtums leisten könne, außerhalb der EU zu bleiben,

12 Stoiber, M./Thurner, P. W.: Der Vergleich von Ratifikationsstrukturen der EU-Mitgliedsländer für intergouvernementale Verträge. Eine Anwendung des Veto-Spieler Konzeptes, in: Mannheimer Zentrum für europäische Sozialforschung, Arbeitspapier 27, 2000, 24. Im Jahrbuch der Europäischen Integration wurden jedoch auch politische Gründe im Vorfeld der Absage identifiziert, da kurz zuvor ein landesweites Referendum zur Abtreibung an der mangelnden Wahlbeteiligung gescheitert war: „Zu groß war die Angst - über alle politischen Parteien hinweg -, dass das Thema ebenfalls aufgrund mangelnder Wahlbeteiligung scheitern könne. Nachdem das Verfassungsgericht die vom Parlament vorgenommene Bündelung der Themen als verfassungswidrig beurteilt hatte, konnte die ganze Frage ohne allzu große politische Peinlichkeit beiseite gelegt werden“ (Jahrbuch der Europäischen Integration 1998, 383). 
um so sein Wohlfahrtssystem zu sichern. ${ }^{13}$ Lediglich im Fall von Zypern, als 2004 der Annan-Plan zur Wiedervereinigung abgelehnt wurde, waren Ressentiments gegenüber dem türkischen Teil Zyperns sowie der ungeklärte Verbleib mehrere tausend Toter im Gefolge der türkischen Invasion von 1974 der Hauptgrund für die Ablehnung, die den Beitritt Nordzyperns zur EU bedeutet hätte.

Mit Norwegen (1972 und 1994), den Niederlanden (2005) und Zypern (2004) gibt es drei Länder, in denen nur abgelehnte Entscheide zu beobachten waren, während sich mit Schweden, Irland, Dänemark, Frankreich und der Schweiz immerhin fünf Länder finden, die sowohl ablehnende als auch zustimmende Volksentscheide aufweisen. Nur mehr eine Minderheit stellt die Gruppe von fünf EU-Ländern dar, in denen es bislang keinerlei Europaabstimmungen gegeben hat (Belgien, Bulgarien, Deutschland und Griechenland). Immerhin 17 EU-Länder haben ausschließlich zustimmende Volksentscheide verabschiedet. In den anderen europäischen Ländern kam es - bis auf eine positive Abstimmung in der Türkischen Republik Nordzypern (2004) - zu keinen weiteren Volksabstimmungen. Die meisten Volksabstimmungen mit europarelevantem Inhalt fanden in der Schweiz statt $(n=10)$.

Inhaltlich lassen sich die Volksabstimmungen weiter differenzieren und systematisieren. ${ }^{14}$ Im Folgenden werden die 54 Volksabstimmungen zunächst nach folgenden vier Themenfeldern klassifiziert (vgl. Abb. 2):

- Beitritt zu bzw. Austritt aus europäischen Organisationen,

- Abstimmung über Europäische Vertragswerke,

- Territoriale Aspekte sowie

- Nationale Verfassungsreformen.

Die meisten Abstimmungen $(n=24)$ gab es über den Beitritt zu bzw. den Austritt aus europäischen Institutionen (z.B. Großbritannien 1975 und Grönland 1982), hauptsächlich zur/aus der EG bzw. der EU, aber auch zum Europäischen Wirtschaftsraum EWR. Die zweitgrößte Gruppe $(n=21)$ der europarelevanten Abstimmungen befasst sich mit europäischen Vertragswerken, wie den Verträgen von Maastricht, Amsterdam, Nizza und Lissabon oder mit dem VVE. Territoriale Abstimmungen sowie nationale Verfassungsreformen, die dem Beitritt zu

13 Siegler-Verlag (Hg.): Archiv der Gegenwart. Verschiedene Jahrgänge, hier: 1994, 39519.

14 Auer, A.: Nationale Volksabstimmungen über die Verfassung von Europa: Erfahrungen, Rechtsgrundlagen, Gefahren und Perspektiven, in: ZSE 4 (2004), 580-596; Shu, M.: Referendums and the Political Constitutionalisation of the EU, in: European Law Journal 14/4 (2008), 423-455. 
europäischen Institutionen mitunter vorgeschaltet sind, machen nur eine Minderheit der Abstimmungen aus.

Abbildung 2: Themenfelder europarelevanter Abstimmungen (bis 2010)

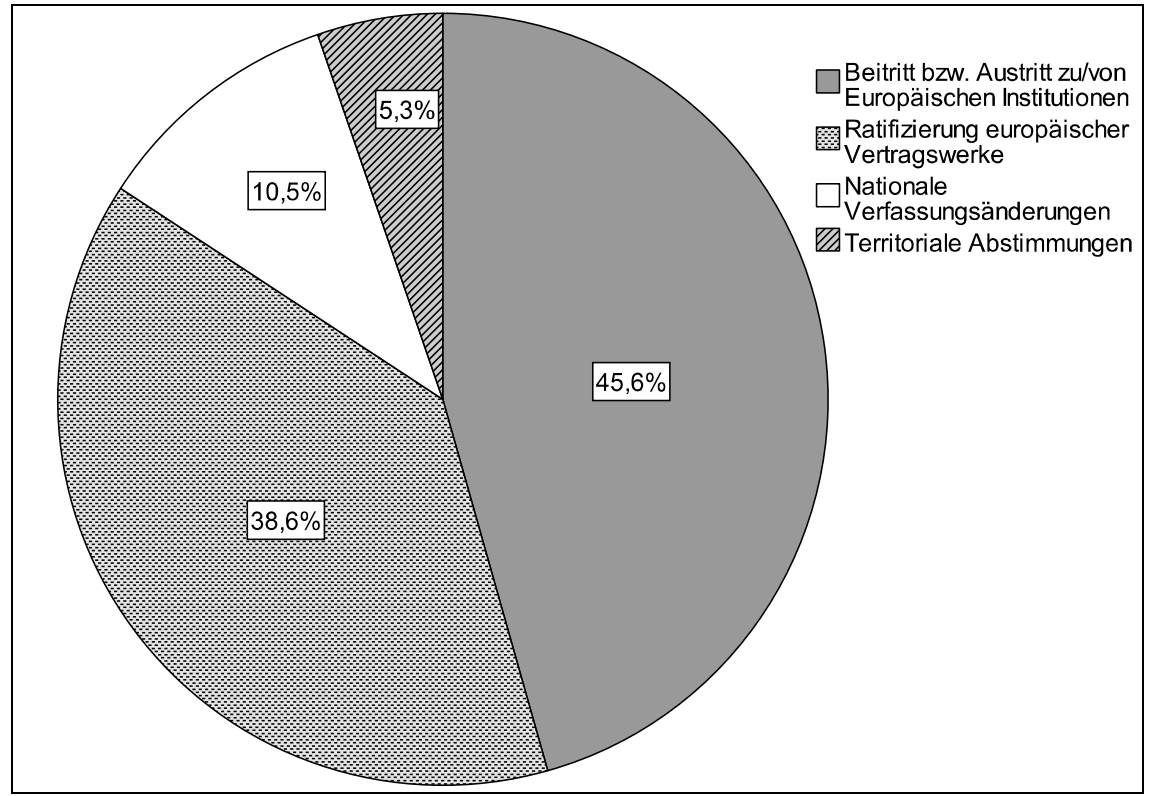

Anmerkungen: Einstufung auf Basis eigener Datenerhebungen und Recherchen, exklusive außereuropäischer und subnationaler Volksabstimmungen sowie der vom Verfassungsgericht Portugals verhinderten Abstimmung zum Amsterdamer Vertrag.

Schließlich kann man die Volksabstimmungen mit Europabezug noch nach ihrem institutionellen Typ differenzieren. Je 22 der 54 Volksabstimmungen waren obligatorische Referenden und Plebiszite (konsultative Referenden). Hinzu treten sechs fakultative Referenden, zwei Volksinitiativen sowie zwei ,ad-hoc“Volksentscheide (Zypern), die von den Vereinten Nationen verantwortet wurden. Jeweils fünf Volksentscheide mit einem negativen Ausgang gab es bei den obligatorischen Referenden und den Plebisziten, ebenso wurden die beiden Volksinitiativen und eine der beiden Zypern-Abstimmungen der VN abgelehnt.

\section{Hypothesen}

Vor dem Hintergrund der aktuellen Herausforderungen für die europäischen Einrichtungen, ablehnenden Volksabstimmungen $\mathrm{zu}$ weitergehenden Integrationsschritten (in den Niederlanden, Frankreich und Irland), der aktuellen Wirt- 
schafts- und Finanzkrise, der Krise des Euro, aber auch angesichts der Diskussion um den Beitritt der Türkei sowie der zentralen gesellschaftlichen Herausforderungen Europas (Migration, demografischer Wandel, soziale Exklusion) ist die Frage „Wer sagt warum nein zu einer vertieften europäischen Integration?“ von weitreichender Relevanz. Die zentrale abhängige Variable zur Beantwortung dieser Frage ist - auf der nationalstaatlichen Aggregatebene - der JaStimmenanteil (in Prozent). Für die empirische Analyse stellt sich die Frage, wie die unterschiedlichen Zustimmungsanteile in den Abstimmungen erklärt werden können. Dabei werden zehn unterschiedliche Hypothesen zur Erklärung herangezogen, deren Wurzeln in unterschiedlichen Theorien der vergleichenden Politikwissenschaft und der Politischen Ökonomie liegen. Mit Hilfe statistischer Analysen soll ihre Tragfähigkeit überprüft werden.

(1) Zunächst ist zu erwarten, dass Länder, die relativ hohe Wohlstandsverluste zu erwarten haben oder Nettozahler in der EU sind, eine größere Wahrscheinlichkeit der Ablehnung von Europaabstimmungen besitzen. Umgekehrt dürfte der Anteil der Ja-Stimmen bei den Ländern höher liegen, die eher arm sind und sich ökonomische Vorteile von einem Beitritt versprechen, etwa über Transfers im Rahmen der EU-Kohäsionsfonds. Diese rent-seeking-Hypothese geht daher funktional von einem negativen Zusammenhang zwischen den Ja-Stimmenanteilen und dem „Wohlstandsabstand“ eines Landes zum OECD-Durchschnitt (gemessen zum Zeitpunkt der Abstimmung) aus, d.h. je ärmer ein Land, desto höher der Ja-Stimmenanteil. Auf der Individualebene steht der homo oeconomicus als Erklärungsmodell hierfür Pate. Dabei geben Präferenzen und Handlungsrestriktionen die Möglichkeiten vor, in denen der homo oeconomicus versucht, auf der Basis rationaler Überlegungen seinen Nutzen zu maximieren. ${ }^{15}$ Individuen wählen demnach so, dass ihnen aus der Wahl ein möglichst großer Nutzen entsteht (Prinzip der Nutzenmaximierung).

(2) Die Diskursthese postuliert, dass die Zustimmung in der Volksabstimmung nachlässt, je stärker zwischen den politischen Akteuren gestritten wird. Bei der Analyse von Hobolt zu den Einflussfaktoren von europäischen Volksabstimmungen auf der Individualebene ist es vor allem die Kampagnenauseinandersetzung, die eine hohe Relevanz erfährt. Ein Faktor für erfolgreiche Volksabstimmungen sind folglich die Überzeugungsstrategien und die Konsensfähigkeit der

15 Kirchgässner, G.: Homo oeconomicus: Das ökonomische Modell individuellen Verhaltens und seine Anwendung in den Wirtschafts- und Sozialwissenschaften. Die Einheit der Gesellschaftswissenschaften (Hg.: M. Albert). 3. Auflage 2008, Tübingen, 1991, 17f. 
Eliten. Dabei wurde die Konflikthaftigkeit des innenpolitischen Diskurses mit $3=$,hoch bzw. umstritten“, $2=$,,mittel bzw. unauffällig“ sowie $1=$,niedrig bzw. Einigkeit" bewertet (mit Abstufungen von 0,5). Als Grundlage dienten Auswertungen des Abstimmungskampfes im Jahrbuch der Europäischen Integration, im Archiv der Gegenwart sowie in der zeitgenössischen bzw. aktuellen journalistischen Berichterstattung in Deutschland sowie teilweise in den Untersuchungsländern.

(3) Die Identitätsthese besagt, dass die Wahrscheinlichkeit einer Zustimmung steigt, je höher die europäische Identität bzw. die Zustimmung zu Europa als Ganzem ist. Große Vorbehalte gegenüber Europa führen dementsprechend zu niedrigeren Zustimmungsanteilen. Diese Identitätsthese kann anhand der in den Eurobarometer-Umfragen gemessenen Zustimmung zu Europa überprüft werden. $^{16}$

(4) Die Partizipationsthese besagt in ihrer ersten Variante, dass die Zustimmung mit einem steigenden Angebot an Abstimmungsmöglichkeiten in einem Land (rules in form) sinkt, weil die Bürger durch viele Abstimmungen ,übersättigt“ sind. Dieser Effekt ist vor allem im „Mutterland der direkten Demokratie“, der Schweiz, zu beobachten. In der zweiten Variante wird die These noch um die faktische Beteiligung an der Abstimmung (rules in use) ergänzt. Datengrundlage sind die beiden zentralen Indikatoren, die im vorangegangenen Abschnitt vorgestellt wurden.

(5) Die second-order-These geht von der Überlegung aus, dass Volksabstimmungen nicht nur zu ihrem eigentlichen Zweck - der Sachabstimmung -, sondern auch als „Abstrafungsinstrument“ insbesondere aufgrund der Innenpolitik genutzt werden. So haben bei den Europawahlen vom Juni 2004 die nationalen Regierungsparteien der damals 25 EU-Länder im Vergleich zur vorhergehenden nationalen Parlamentswahl einen durchschnittlichen Stimmenverlust (gegenüber nationalen Parlamentswahlen) von 11,5 Prozentpunkten erfahren müssen. Überprüft wird diese These anhand des zeitlichen Abstands der Abstimmung von der vorhergehenden nationalen Parlamentswahl. Ein solcher Abstrafungseffekt lässt sich in Deutschland beispielsweise bei Europa- und Landtagswahlen gut beobachten, die daher auch als second-order-Wahlen gelten können. ${ }^{17}$

17 Reif, K./Schmitt, H.: Nine second order national elections. A conceptual framework for the analysis of European election results, in: European Journal of Political Research 8/1 (1980), 3-44. 
(6) Ein wichtiger Untersuchungsgegenstand der Politikwissenschaft ist, wie ökonomische Ergebnisse und soziale Problemlagen die Wünsche von Wählern beeinflussen. Demokratische Systeme legitimieren sich in hohem Maße über ihren politischen output. ${ }^{18}$ Folglich wird einem System umso mehr Legitimation entgegengebracht, je mehr Bürger von seinen Leistungen - z.B. in Form einer niedrigen Arbeitslosigkeit oder einer geringen Inflationsrate - profitieren. Umgekehrt lässt sich damit als Problemdruckthese formulieren, dass die Zustimmung mit wachsendem sozioökonomischem Druck zurückgeht.

(7) Im Sinne der Autonomiethese wäre zu erwarten, dass Volksabstimmungen mit Europabezug in föderalen und ethnisch fragmentierten Systemen eher abgelehnt werden. Das Streben nach Autonomie und Eigenständigkeit und der Wunsch nach Bewahrung kultureller Eigenständigkeit könnte nämlich auch mit einem diffusen Unbehagen vor Europa einhergehen.

(8) Einen weiteren Erklärungsansatz könnte die Parteiendifferenzthese liefern. ${ }^{19}$ Dabei wird überprüft, ob die Zustimmung unter Links- oder Rechtsregierungen höher ist. Generell lassen sich sowohl für Links- als auch für Rechtsparteien Argumente finden, die jeweils eine stärkere oder auch schwächere Unterstützung für Europa unterstellen könnten. So könnte der Internationalismus von Linksparteien grundsätzlich eine positive Wirkung entfalten, während der Protektionismus bei deren Kernklientel hingegen eher negativ wirkt. Und bürgerliche Parteien (insbesondere Christdemokraten) haben lange die europäische Integration vorangetrieben, während der Kernklientel bürgerlicher Parteien Angst vor Überfremdung unterstellt werden, was wiederum negativ auf die Ja-Stimmenanteile wirken dürfte. Insofern wird die These zunächst „,ungerichtet“ formuliert.

(9) Im Rahmen der institutionalistischen These wird vermutet, dass der Anteil von Ja-Stimmen sowohl mit der Anzahl der Parteien in der Regierung als auch mit der politischen Stabilität eines Landes zunimmt. Daneben wurde noch getestet, ob die Mitgliedschaft in der EU an sich oder ihre konkrete Dauer einen Effekt haben.

(10) Schließlich argumentiert die xenophobische These, dass in Ländern mit einem hohen Ausländeranteil (zum Abstimmungszeitpunkt) die Zustimmung zur europäischen Integration - unter sonst gleichen Umständen - niedriger sein wird.

18 Scharpf, F.W.: Demokratietheorie zwischen Utopie und Anpassung. Konstanz, 1970.

19 Castles, F.G. (Hg.): The Impact of Parties. Politics and Policies in Democratic Capitalist States, London, 1982; Schmidt, M.G.: Wohlfahrtsstaatliche Politik unter bürgerlichen und sozialdemokratischen Regierungen, Frankfurt a.M./New York, 1982. 


\section{Befunde des internationalen Vergleichs}

Die nachfolgende Analyse basiert auf den Daten von maximal 50 nationalstaatlichen Volksabstimmungen mit europapolitischem Inhalt im Zeitraum von 1972 bis 2009. Zentral ist dabei das Erkenntnisinteresse mit Blick auf Strukturen und Gemeinsamkeiten über die untersuchten Fälle hinweg. Nationalstaatliche Kontexte werden nach Möglichkeit und entsprechend der formulierten Hypothesen auch durch Variablen modelliert (z.B. Föderalismus, EU-Mitgliedschaft, Ausländeranteil). Jedoch bleiben spezifische nationale Kontexte durch diesen Ansatz unterbelichtet oder werden nur punktuell berücksichtigt, wie etwa die Position Luxemburgs als zentraler Profiteur der EU (Luxemburg-dummy-Variable). Da jede Abstimmung ihren eigenen Abstimmungskampf ${ }^{20}$ und Kontext (z.B. innenpolitische Krisen oder außenpolitische Entwicklungen) besitzt, sind Länderidiosynkrasien naturgemäß groß. Hier ist jedoch das Streben nach der Identifikation von Gemeinsamkeiten, Strukturen und Muster forschungsleitend.

Durch die Messung zum Abstimmungszeitpunkt kommt es bei einigen Variablen $\mathrm{zu}$ „fehlenden Werten“. So liefern die Eurobarometer-Umfragen (EB), die als Datenbasis für die Frage zur Zustimmung zu Europa (= europäische Identität) dienen, in der Regel nur Daten für die EU-Mitgliedsländer (bzw. für die Beitrittsländer). Seit 1974 wurden bis Ende 2010 insgesamt 74 solcher Befragungen (zweimal jährlich) durchgeführt. ${ }^{21}$ Dabei wurde der Wert erhoben, der dem Abstimmungstermin jeweils am nächsten liegt. Für die erste Europa-Abstimmung 1972 in Frankreich wurde daher der Wert der ersten Eurobarometerbefragung, durchgeführt im Herbst 1973, verwendet, ansonsten ist jedoch eine große zeitliche Nähe zur Abstimmung gegeben. Ebenso fehlen für den Ausländeranteil einige Werte.

Für die empirischen Regressionsanalysen wurde in allen acht Gleichungen der Tab. 2 und 3, mit Ausnahme von Gleichung 1, ein Basismodell aus den drei zentralen Erklärungsfaktoren verwendet: der relativen Wohlstandsposition eines

20 Hobolt, S.B., a.a.O.

21 Die hier verwendete Operationalisierung basiert auf der Zustimmungskategorie der fortlaufend erhobenen, standardisierten Frage: ,Ist die Mitgliedschaft (ihres Landes) in der Europäischen Union Ihrer Meinung nach ... (a) ...eine gute Sache? (b) ...eine schlechte Sache? (c) ...weder gut noch schlecht?" Für die Schweiz wurden die Antworten auf die von der Gesellschaft für praktische Sozialforschung GFS Bern im Rahmen des Europa-Barometer Schweiz gestellte Frage herangezogen, ob die befragte Person einem EU-Beitritt zustimmen würde oder nicht; vgl. Longchamp, C.: Schub Richtung Integration. Hauptergebnisse aus dem neuesten Bericht zum Europa-Barometer Schweiz, Sommer 2004, Bern, 2004; Longchamp, C.: Offen auf die Zukunft zugehen. Hauptergebnisse aus dem neuesten Bericht zum Europa-Barometer Schweiz, Winter 2005, Bern, 2005. 
Landes (gemessen als Wohlstandsabstand zum EU-Durchschnitt), der Intensität des politischen Diskurses sowie der Zustimmung zu Europa. Für die einzelnen Hypothesen wurden dann jeweils die einzelnen Variablen ,hinzugespielt“. Gleichung 1, die auf der maximal möglichen Fallzahl beruht, zeigt die Befunde nur für die ersten beiden Faktoren.

Die rent-seeking-Hypothese wird eindrucksvoll in allen acht Gleichungen bestätigt. Sowohl die rational-choice-Theorie als auch das Columbia-Modell von Lazarsfeld $^{22}$ können hier als theoretische Grundlagen herangezogen werden. Sie zeigen an, dass die Abstimmungsentscheidung vor allem aus wirtschaftlichen Überlegungen heraus getroffen wird. Die Bevölkerung in reichen Ländern wird bei weiteren Integrationsschritten - so zumindest die Perzeption der Abstimmenden - von Wohlfahrtsverlusten bedroht. Dies gilt etwa für Länder, die einem Beitritt zu europäischen Institutionen (zumindest weitgehend) widerstanden, wie etwa Norwegen und die Schweiz. Aber auch die Bürger in Staaten, die sich bereits innerhalb der EU befinden, befürchten angesichts neuer Beitrittsländer und weiterer Integrationsschritte einen Abbau von Sozialstandards, weitere Marktöffnungen und den Zuzug billigerer Arbeitskräfte. Beispiele hierfür waren die negativen Voten in Dänemark 1992 sowie in Frankreich und den Niederlanden 2005. Aber auch die Angst vor dem Verlust von Hilfszahlungen aus Brüssel prägte in manchen Ländern die Abstimmungsentscheidung. Beispiele dafür finden sich in den Abstimmungsentscheidungen in Irland 2001 und 2008 sowie in der Abstimmungsdiskussion 2005 in Spanien. Für die Bürger der - in der Regel - weniger wohlhabenden Beitrittskandidaten liegt die Interessenlage naturgemäß anders, da sie sich ökonomische Umverteilung, Transfers und Marktzugang versprechen. Die Luxemburg-dummy-Variable ${ }^{23}$ ist im Übrigen auch eine Größe, die perfekt zu dieser Hypothese passt. Die Analysergebnisse zeigen, dass das Resultat - unter sonst gleichen Umständen - bei der Luxemburgischen Volksabstimmung um rund 32 Prozentpunkte über den Erwartungen liegt.

Auch die Identitätsthese ist in fast allen Gleichungen, bis auf Gleichung 5 und 8, zumindest auf dem 5-Prozent-Niveau signifikant. Dabei besitzt die Variable das erwartete Vorzeichen. Dies bedeutet: Je höher die Zustimmung zu Europa in

22 „A person thinks, politically, as he is, socially“; Lazarsfeld, P.: People's Choice: How the Voter Makes Up His Mind in a Presidential Campaign, 3. Aufl., New York, 1965: 27.

23 Diese Variable wurde in den nachfolgenden Gleichungen aufgrund hoher Multikollinearität ausgeschlossen. 
einem Land ist, desto größer ist auch die Zustimmung in Volksabstimmungen mit europapolitischem Inhalt.

Tabelle 2: Bestimmungsfaktoren des Erfolgs von Volksabstimmungen mit Europabezug (Abhängige Variable: Prozentanteil der Ja-Stimmen)

\begin{tabular}{|c|c|c|c|c|}
\hline Gleichung & (1) & (2) & (3) & (4) \\
\hline Konstante & $\begin{array}{c}90,291 \\
(15,442)^{* * * *}\end{array}$ & $\begin{array}{l}71,581 \\
(6,523)^{* * *}\end{array}$ & $\begin{array}{l}57,125 \\
(4,287)^{* * *}\end{array}$ & $\begin{array}{l}46,534 \\
(2,438)^{* *}\end{array}$ \\
\hline $\begin{array}{l}\text { BIP pro Kopf-Differenz. zum } \\
\text { EU-Durchschnitt } \\
\text { (Abstimmungszeitpunkt) }\end{array}$ & $\begin{array}{l}-0,001 \\
(-3,307)^{* * *}\end{array}$ & $\begin{array}{c}-0,001 \\
(-3,818)^{* * * *}\end{array}$ & $\begin{array}{l}-0,001 \\
(-4,304) * * *\end{array}$ & $\begin{array}{l}-0,001 \\
(-4,245) * * *\end{array}$ \\
\hline $\begin{array}{l}\text { Intensität des innenpolitischer } \\
\text { Diskurses ( } 1 \text { bis } 3,3=\text { hoch })\end{array}$ & $\begin{array}{l}-13,795 \\
(-5,263) * * *\end{array}$ & $\begin{array}{l}-13,047 \\
(-4,281) * * *\end{array}$ & $\begin{array}{l}-9,895 \\
(-3,079) * * *\end{array}$ & $\begin{array}{l}-9,812 \\
(-3,007) * * *\end{array}$ \\
\hline $\begin{array}{l}\text { Zustimmung zu Europa zum } \\
\text { Abstimmungszeitpunkt } \\
\text { (Quelle: EB) }\end{array}$ & & $\begin{array}{c}0,307 \\
(2,418)^{* *}\end{array}$ & $\begin{array}{l}0,292 \\
(2,266) * *\end{array}$ & $\begin{array}{l}0,318 \\
(2,365)^{* *}\end{array}$ \\
\hline Luxemburg-dummy-Variable & & & $\begin{array}{l}32,002 \\
(2,020)^{*}\end{array}$ & \\
\hline $\begin{array}{l}\text { rules in form (additiver Index, } \\
1 \text { bis } 5 \text {; dabei: } 5=\text { viel Partizi- } \\
\text { pationsmöglichkeit) }\end{array}$ & & & $\begin{array}{c}3,033 \\
(1,619)\end{array}$ & $\begin{array}{c}3,559 \\
(1,943) *\end{array}$ \\
\hline $\begin{array}{l}\text { dummy-Variable für rules in } \\
\text { use }(1=\text { hohe Zahl an Volks- } \\
\text { abstimmungen })\end{array}$ & & & $\begin{array}{c}0,265 \\
(0,059)\end{array}$ & \\
\hline $\begin{array}{l}\text { Abstimmungsbeteiligung in } \\
\text { Prozent (Partizipationsrate) }\end{array}$ & & & & $\begin{array}{c}0,076 \\
(0,549)\end{array}$ \\
\hline $\begin{array}{l}\text { Tage bis zur nächsten Parla- } \\
\text { mentswahl (second-order- } \\
\text { Hypothese) }\end{array}$ & & & & $\begin{array}{c}0,004 \\
(0,841)\end{array}$ \\
\hline Adj. $R^{2}$ & 0,493 & 0,554 & 0,591 & 0,599 \\
\hline F-Statistik & $24,805 * * *$ & $18,425^{* * * *}$ & $11,132^{* * * *}$ & $11,220 * * *$ \\
\hline $\mathrm{N}$ & 50 & 43 & 43 & 42 \\
\hline
\end{tabular}

Anmerkungen: Die Werte sind die unstandardisierten Regressionskoeffizienten; t-Statistik in Klammern: $*=$ signifikant auf $10 \%$-Niveau, $* *=$ signifikant auf 5\%-Niveau, $* * *=$ signifikant auf $1 \%$ Niveau (zweiseitige Fragestellung beim Hypothesentest). EB = Eurobarometer (verschiedene Erhebungen). In keiner Gleichung liegt Multikollinearität vor (VIF-Wert ist stets kleiner als 2,0).

Die Messung sowohl für die rules in form als auch die rules in use findet keine überzeugende empirische Unterstützung. Lediglich für das institutionalisierte Partizipationsangebot kann ein schwach signifikanter Einfluss ausgemacht werden. Eine hohe Zahl an Volksabstimmungen erweist sich als insignifikant und 
damit als nicht erklärungskräftig. Zudem haben weitere Untersuchungen gezeigt (nicht in Tab. 2 und 3 enthalten), dass eine hohe Wahlbeteiligung sich nicht negativ auf den Anteil der Ja-Stimmen einer europarelevanten Abstimmung auswirkt.

Der Aspekt der Abstrafung bzw. Disziplinierung von Regierungen kommt in der second-order-These zum Ausdruck. Zwei Operationalisierungen wurden zu ihrer Überprüfung herangezogen: Einmal der zeitliche Abstand (gemessen in Tagen) seit der letzten nationalen Wahl und zum anderen der zeitliche Abstand zu den kommenden Wahlen, in Gleichung 4 von Tab. 2 verwendet. Letztere Variable ist zwar bei aktuellen Abstimmungen mit einer gewissen Unsicherheit behaftet, da vorgezogene Neuwahlen nicht ausgeschlossen werden können und nicht alle Wahltermine bereits auf einen genauen Tag festgelegt sind. Keine der beiden Variablen war jedoch signifikant, was den Aspekt der Abstrafung empirisch nicht unterstützt.

Die Problemdruckthese wurde mit den Standardvariablen Arbeitslosigkeit, Inflation, Wirtschaftswachstum und außenwirtschaftliche Verflechtung (nicht in Tab. 3) gemessen. So hat etwa Hug (2002) gezeigt, dass die Zustimmung zur europäischen Integration dort höher ist, wo auch die Handelsverflechtung besonders groß ist, was sich jedoch mit den vorliegenden Daten, die auf einer deutlich höheren Zahl an Abstimmungen beruht, nicht nachweisen lässt. Um den Problemdruck zu erfassen, wurde eine aggregierte Variable aus der Addition der Arbeitslosenquote und der Inflationsrate abzüglich des Wirtschaftswachstums gebildet (der sogenannte misery-Index). Dieser Faktor (vgl. Gleichung 5 in Tab. 3) erweist sich als hoch signifikant und erklärungskräftig. Allerdings sinken mit ansteigendem Problemdruck nicht die Zustimmungsanteile, sondern liegen ceteris paribus - höher. Offenbar wird die EU hier eher als Problemlöser für ökonomische Schwierigkeiten begriffen, denn als Problemerzeuger.

Auch die Variablen der Autonomiethese, der Parteiendifferenzthese sowie der institutionalistischen Hypothese schneiden in der Erklärung der Zustimmungsquote bei europarelevanten Volksabstimmungen schlecht ab (Gleichung 6, 7, 8 in Tab. 3). Weder eine föderale Staatsstruktur noch die Stärke von Linksparteien oder die Stärke von bürgerlichen Parteien, die Zahl der Parteien an der Regierung, die EU-Mitgliedschaft oder die Dauer der EU-Mitgliedschaft haben einen signifikanten Einfluss.

Schließlich zeigen die Befunde aus Tab. 3 (Gleichung 8), dass der Ausländeranteil keinen Einfluss auf die Ja-Stimmenanteile besitzt. Dies bedeutet zwar nicht, 
dass die xenophobische These generell keine Rolle spielt, ein signifikanter Einfluss kann jedoch nicht nachgewiesen werden.

Tabelle 3: Bestimmungsfaktoren des Erfolgs von Volksabstimmungen mit Europabezug (Abhängige Variable: Prozentanteil der Ja-Stimmen)

\begin{tabular}{|c|c|c|c|c|}
\hline Gleichung & (5) & (6) & (7) & (8) \\
\hline Konstante & $\begin{array}{l}70,970 \\
(7,172)^{* * *}\end{array}$ & $\begin{array}{l}66,218 \\
(3,815)^{* * * *}\end{array}$ & $\begin{array}{l}56,582 \\
(3,631)^{* * *}\end{array}$ & $\begin{array}{l}71,794 \\
(4,937)^{* * * *}\end{array}$ \\
\hline $\begin{array}{l}\text { BIP pro Kopf-Differenz. zum } \\
\text { EU-Durchschnitt } \\
\text { (Abstimmungszeitpunkt) }\end{array}$ & $\begin{array}{l}-0,001 \\
(-3,380) * * *\end{array}$ & $\begin{array}{l}-0,001 \\
(-3,323)^{* * * *}\end{array}$ & $\begin{array}{l}-0,001 \\
(-3,460) * * *\end{array}$ & $\begin{array}{l}-0,001 \\
(-2,908) * * *\end{array}$ \\
\hline $\begin{array}{l}\text { Intensität des innenpolitischer } \\
\text { Diskurses ( } 1 \text { bis } 3,3=\text { hoch) }\end{array}$ & $\begin{array}{l}-12,574 \\
(-4,452) * * *\end{array}$ & $\begin{array}{c}-13,166 \\
(-4,101)^{* * *}\end{array}$ & $\begin{array}{c}-12,491 \\
(-3,809)^{* * * *}\end{array}$ & $\begin{array}{c}-13,223 \\
(-3,928)^{* * *}\end{array}$ \\
\hline $\begin{array}{l}\text { Zustimmung zu Europa zum } \\
\text { Abstimmungszeitpunkt } \\
\text { (Quelle: EB) }\end{array}$ & $\begin{array}{l}0,206 \\
(1,724) *\end{array}$ & $\begin{array}{c}0,320 \\
(2,114)^{* *}\end{array}$ & $\begin{array}{c}0,382 \\
(2,373) * *\end{array}$ & $\begin{array}{c}0,268 \\
(1,723)^{*}\end{array}$ \\
\hline $\begin{array}{l}\text { misery-Index zum Abstim- } \\
\text { mungszeitpunkt (=ALQ + CPI } \\
\text { - BIP-Wachstum) }\end{array}$ & $\begin{array}{l}0,683 \\
(2,345) * *\end{array}$ & & & \\
\hline $\begin{array}{l}\text { Föderalistisches Land } \\
(0=\text { zentralistisch; } 1 \text { = föderal })\end{array}$ & & $\begin{array}{l}0,706 \\
(0,135)\end{array}$ & & \\
\hline $\begin{array}{l}\text { Parteipolitische Zusammenset- } \\
\text { zung der Regierung } \\
\text { (t, } 1 \text { bis } 5 \text {, dabei: } 5=\text { links) }\end{array}$ & & $\begin{array}{c}1,660 \\
(1,222)\end{array}$ & $\begin{array}{c}2,258 \\
(1,532)\end{array}$ & \\
\hline $\begin{array}{l}\text { EU-Mitgliedschaft (Abstim- } \\
\text { mungszeitpunkt mit } 1=\text { EU- } \\
\text { Mitglied) }\end{array}$ & & & $\begin{array}{l}-0,982 \\
(-0,195)\end{array}$ & \\
\hline $\begin{array}{l}\text { Anzahl der Parteien an der } \\
\text { Regierung } \\
\text { (t = Abstimmungszeitpunkt) }\end{array}$ & & & $\begin{array}{l}1,662 \\
(0,947)\end{array}$ & \\
\hline $\begin{array}{l}\text { Ausländeranteil (Prozentanteil } \\
\text { der im Ausland Geborenen, t) }\end{array}$ & & & & $\begin{array}{c}0,127 \\
(0,365)\end{array}$ \\
\hline Adj. $R^{2}$ & 0,606 & 0,549 & 0,551 & 0,542 \\
\hline F-Statistik & $16,776^{* * * *}$ & $11,217^{* * * *}$ & $9,574 * * * *$ & $11,952^{* * * *}$ \\
\hline $\mathrm{N}$ & 42 & 43 & 43 & 38 \\
\hline
\end{tabular}

Anmerkungen: Die Werte sind die unstandardisierten Regressionskoeffizienten; t-Statistik in Klammern: $*=$ signifikant auf dem 10\%-Niveau, $* *=$ signifikant auf dem 5\%-Niveau, $* * *=$ signifikant auf dem 1\%-Niveau (zweiseitige Fragestellung beim Hypothesentest). Mit folgenden Ausnahmen liegt in keiner Gleichung Multikollinearität vor (VIF-Wert ist stets kleiner als 2,0): Gleichung 7 für den EU-Anteil (VIF = 2,003) sowie in Gleichung 8 für den Reichtumsabstand zum EU-Mittel (VIF = 2,056) und den Ausländeranteil ( $\mathrm{VIF}=2,144)$. 


\section{Zusammenfassung und Ausblick}

Volksabstimmungen mit Europabezug sind für Regierungen eine riskante Angelegenheit. Dies hat sich nach dem Scheitern des Vertrages über die Verfassung Europas (VVE) in Frankreich und den Niederlanden 2005 gezeigt. Wollten diesen Vertrag noch insgesamt 10 Länder direktdemokratisch ratifizieren (sechs Volksabstimmungen wurden nach dem Scheitern der beiden Abstimmungen verschoben bzw. blieben offen), war es beim Nachfolgewerk - dem Vertrag von Lissabon - nur noch Irland, das ein obligatorisches Referendum anberaumte. Auch dieses scheiterte, bevor die Iren 2009 in einer zweiten Abstimmung (wie bereits 2001 und 2002 beim Vertrag von Nizza) das „Ja“ lieferten. Der vorliegende Beitrag hat auf Basis sämtlicher bisher abgehaltener Volksabstimmungen jene Faktoren herausgearbeitet, die den Ja-Stimmenanteil solcher direktdemokratischer Entscheide mit Europabezug beeinflussen. Die zentralen Befunde werden in Tab. 4 nochmals zusammengefasst und anhand ihrer empirischen Gültigkeit bewertet.

Insgesamt wurde bei den Volksabstimmungen mit europarelevantem Inhalt deutlich, dass die Wählerschaft in Europa in ihrem Abstimmungsverhalten sehr bewusst ökonomische Überlegungen zugrunde legt: Der homo oeconomicus war ein starker Motor für die positiven Integrationsentscheidungen in Ost- und Mitteleuropa. Aber auch in Luxemburg kann die hohe Zustimmungsrate auf rentseeking-Überlegungen zurückgeführt werden. Ebenso neigen überdurchschnittlich wohlhabende Länder dazu, sich eher abzuschotten, um Umverteilungsansprüche zu vermeiden.

Beachtlich ist auch die Bedeutung des innenpolitischen Diskurses als Einflussfaktor für die Erfolgswahrscheinlichkeiten von Volksabstimmungen: Je konfliktiver die politische Auseinandersetzung, desto geringer ist die Erfolgswahrscheinlichkeit für die Annahme. Die wachsende Unpopularität Europas vor dem Hintergrund der Finanz-, Schulden- und Eurokrise macht es für politische Unternehmer „rentabler“, im Falle einer solchen Abstimmung diese Konfliktlinie auszubeuten. Daher wird es zukünftig schwieriger sein, einen Elitenkonsens und damit eine niedrige Konfliktintensität - herzustellen.

Der dritte entscheidende Faktor ist die Zustimmung zu Europa. Diese ist - so die zweimal jährlich erhobenen Daten des Eurobarometers - über die Jahre im Schwinden begriffen. Insofern wird es insgesamt schwerer werden, Volksabstimmungen über Europa erfolgreich zu gestalten. 
Tabelle 4: Ergebnisse der multivariaten Analyse zur Erklärung der Ja-Stimmenanteile in Volksabstimmungen mit Europabezug

\begin{tabular}{|c|c|c|}
\hline These & $\begin{array}{c}\text { Erklärung / Interpretation: } \\
\text { Die Ja-Stimmenanteile sind umso größer, ... }\end{array}$ & $\begin{array}{l}\text { Signifikante } \\
\text { Bestätigung der } \\
\text { These? }\end{array}$ \\
\hline (1) rent-seeking-These & ... je ärmer ein Land ist. & $\mathrm{Ja}$ \\
\hline (2) Diskursthese & $\begin{array}{l}\text {... je konsensualer der Diskurs im Abstim- } \\
\text { mungskampf ist. }\end{array}$ & $\mathrm{Ja}$ \\
\hline (3) Identitätsthese & ... je größer die Zustimmung zu Europa ist. & $\mathrm{Ja}$ \\
\hline (4) Partizipationsthese & $\begin{array}{l}\text { (a)... je größer das Partizipationsangebot ist. } \\
\text { (b)... je mehr Volksabstimmungen stattfinden. } \\
\text { (c)... je niedriger die Stimmbeteiligung ist. }\end{array}$ & $\begin{array}{l}\text { (a) Schwach } \\
\text { (b) Nein } \\
\text { (c) Nein }\end{array}$ \\
\hline (5) second-order-These & $\begin{array}{l}\text {... je kürzer der Abstand zu der nächsten } \\
\text { Parlamentswahl ist. }\end{array}$ & Nein \\
\hline (6) Problemdruckthese & $\begin{array}{l}\text {... je geringer der ökonomische Problem- } \\
\text { druck (z.B. die Arbeitslosigkeit) ist. }\end{array}$ & $\begin{array}{l}\text { Nein; umgekehr- } \\
\text { ter Zusammen- } \\
\text { hang gültig }\end{array}$ \\
\hline (7) Autonomiethese & ... je zentralistischer ein Staat verfasst ist. & Nein \\
\hline (8) Parteiendifferenzthese & $\begin{array}{l}\text { (a)... je stärker Linksparteien an der Regierun- } \\
\text { beteiligt sind. } \\
\text { (b)... je stärker bürgerliche Parteien an der } \\
\text { Regierung beteiligt sind. }\end{array}$ & $\begin{array}{l}\text { (a) Nein } \\
\text { (b) Nein }\end{array}$ \\
\hline $\begin{array}{l}\text { (9) Institutionalistische } \\
\text { These }\end{array}$ & $\begin{array}{l}\text { (a)... je mehr Parteien an der Regierung sind. } \\
\text { (b)... je kürzer ein Land EU-Mitglied ist. }\end{array}$ & $\begin{array}{l}\text { (a) Nein } \\
\text { (b) Nein }\end{array}$ \\
\hline $\begin{array}{l}\text { (10) Xenophobische } \\
\text { These }\end{array}$ & ... je niedriger der Ausländeranteil & Nein \\
\hline
\end{tabular}

Anmerkung: Einstufung auf Basis der Regressionsanalysen der Tab. 2 und 3 und der im Text erwähnten Zusatzauswertungen.

„Positiv“ wirkt jedoch ein hoher Problemdruck - ein Spezialfall der rentseeking-Hypothese. Je schlechter die ökonomische Lage, desto höher die $\mathrm{Zu}$ stimmung. Auch dies war in der aktuellen Wirtschafts- und Finanzkrise gut sichtbar, als zum Höhepunkt 2009 in der Schweiz eine überraschend hohe $\mathrm{Zu}$ stimmung zu Europa zu beobachten war, insbesondere als ein Bankrott der Schweizer Großbank UBS nicht auszuschließen war - und Europa hier Rettung versprach.

Die anderen überprüften Thesen blieben dagegen weitgehend insignifikant. Es konnte weder ein parteipolitischer Einfluss, ein institutioneller Einfluss noch die Autonomiethese (Föderalismus) bestätigt werden. Auch die Abstrafungsthese, die Xenophobiethese sowie die Partizipationsthese blieben, bis auf einen geringen Effekt der rules in form, ohne Bedeutung.

Obwohl die Zurückhaltung der Mitgliedstaaten mit Blick auf direktdemokratische Ratifikationsverfahren beim Vertrag von Lissabon deutlich wurde, dürften 
sich künftige Integrationsschritte aufgrund der maßgeblich erweiterten Mitspracherechte der Völker Europas als politisch schwieriger erweisen (vgl. Tab. 1). Mehrere Staaten haben inzwischen angekündigt, weitere Beitritte zur EU dem Volk vorzulegen. In Frankreich wurde sogar die Verfassung geändert (Art. 85 (5) gem. Verfassungsgesetz Nr. 2008-724 vom 23. Juli 2008): „Jeder Gesetzentwurf, der zur Ratifizierung eines Vertrages über den Beitritt eines Staates zur Europäischen Union ermächtigt, wird vom Präsidenten der Republik zum Volksentscheid gebracht." Dies kann nur durch eine 3/5-Mehrheit in beiden Kammern als Alternativform der Ratifizierung vermieden werden. Dass sich die Direktdemokratie damit zu einem veritablen Vetopunkt und der demos zu einem bedeutendem Vetospieler entwickeln, ist nicht zu übersehen. 